\title{
miR-378d regulates polyploidization and malignant phenotype of tumor cells through AKT and RhoA
}

Jie Peng ${ }^{1,2 \#}$, Susu Shi ${ }^{1 \#}$, Juan $\mathrm{Yu}^{1,3 \#}$, Jianli Liu ${ }^{1}$, Haixiang $\mathrm{Wei}^{4}$, Haixia Song ${ }^{4}$, Shaoqiang Wang ${ }^{4}$, Daolu Guo ${ }^{1}$, Zhejie $\mathrm{Li}^{1}$, Shujin $\mathrm{He}^{1}$, Lei $\mathrm{Li}^{1}$, Hongyan Zhang ${ }^{1}$, Zhizhen Yan ${ }^{1}$, Ran Zhao ${ }^{1}$, Yukun Liu ${ }^{1}$, Yanrong Liu ${ }^{1}$, Junjun $\mathrm{Li}^{5}$, Renya Zhang ${ }^{1}$, Wei Wang $^{1 *}$

1. Department of Pathology, Affiliated Hospital of Jining Medical University, Jining Medical University, Jining, Shandong 272029, China

2. Department of Pathology, Zibo Central Hospital, Zibo, Shandong 255036, China.

3. Department of Pathology, The First People's Hospital of Xuzhou, Xuzhou, Jiangsu 221106, China

4. Department of Thoracic Surgery, Affiliated Hospital of Jining Medical University, Jining Medical University, Jining, Shandong 272029, China

5. Cancer Research Institute and School of Basic Medical Sciences, Central South University, Changsha, Hunan 410078, China

\#: These authors contributed equally to this work.

*: Correspondence to: Wei Wang, Department of Pathology, Affiliated Hospital of Jining Medical University, 89 Guhuai Road, Jining, Shandong 272029, China. Tel: 86-537-2903394; Fax: 86-5372213030; E-mail: weiwangphd@163.com

\section{Simple Summary:}

Chemo-resistance of ESCC is a difficult problem in the current treatment. After drug resistance, esophageal cancer is prone to recurrence and metastasis, and polyploid tumor cells are the focus of the current research. We found a significant decrease in miR-378d expression in paclitaxel resistance polyploid cells and determined that reduced miR-378d expression promotes tumor polyploid production. miR-378d achieves the regulation and malignant phenotype of polyploid tumor cell phenotypes by regulating AKT and RhoA signals. These results demonstrate for the first time the importance of miR-378d in the malignant progression of esophageal cancer and the emphasis that the combination of chemotherapy against polyploid inhibition of tumor cells may be an effective strategy for the treatment of esophageal cancer.

\section{Abstract:}


Studies have shown that stress such as hypoxia, chemotherapy, radiotherapy can lead to polyploidization of tumor cells, which play an important role in tumor heterogeneity and malignant phenotype. Paclitaxel (PTX) treatment promoted polyploid cancer cells (PCCs) formation, and miR-378d is sharply reduced in PCCs of esophageal squamous cell carcinomas (ESCC) cells, but miR-378d participation PCCs formation and the impact on the biological behavior of ESCC remains unclear. We analyzed the PCCs formation and biological behavior of ESCC cells in vivo and in vitro, and the related proteins regulated by miR-378d. Results showed that miR-378d expression was associated with good prognosis in ESCC patients. miR-378d inhibition promoted PCCs formation, heterogenicity, chemo-resistance, monoclonal formation, EMT, migration, invasion, stemness and metastasis of ESCC cells. miR-378d can target downregulated AKT1, and inactivating the AKT- $\beta$-catenin signaling pathway, miR$378 \mathrm{~d}$ and AKT can also regulated RhoA expression. AKT and RhoA regulated polyploidization and depolyploidization. Therefore, miR-378d expression is a good prognostic factor of ESCC patients and regulates polyploidization and malignant phenotype of tumor cells through AKT and RhoA.

Keywords: miR-378d; ESCC; AKT; RhoA; polyploidization

\section{Introduction}

Great progress has been made in the diagnosis and treatment of ESCC in recent years, but ESCC patients' five-year survival rate remains low and no effective targeted therapy is available ${ }^{[1]}$. Chemotherapy, adjuvant therapy, and radiotherapy are still the main treatments for ESCC, particularly for patients in advanced stages. Acquired chemoresistance is also a frequent cause of treatment failure, and it leads to local recurrence and metastasis ${ }^{[2,3]}$.

Polyploid cancer cells (PCCs) are a new development in recent years, it is as intermediate products of genomic instability. PCCs and diploid cancer cells (DCCs) were significantly different in morphology, size, chromosomal abnormalities, tumorigenicity, anti-radiotherapy, and chemoresistance ${ }^{[4]}$. Zhang Shiwu et al ${ }^{[5]}$ defined the ratio of nuclear volume to diploid tumor nucleus $\geq 3$ cells as polyploid giant cancer cells (PGCCs). Although the pre-chemotherapy specimens had relatively homogeneous cells, the number of PGCCs and giant singular nuclei in the post-chemotherapy specimens increased ${ }^{[6]}$. PGCCs with budding have been found in $27.45 \%$ highly 
differentiated, 50\% moderately differentiated and $90.20 \%$ poorly differentiated colorectal cancers, furthermore, the accuracy of predicting lymph node metastasis was $85.71 \%(18 / 21)$ that PGCCs with budding emerged in the stroma of ovarian cancer ${ }^{[7]}$.

Paclitaxel (PTX) is a first-line chemotherapy drug for ESCCs, and can stabilize microtubule polymers to block cell cycle during the G2-M phase, hindering the development of mitosis, thus forming PGCCs ${ }^{[6,8]}$. Studies have reported that AKT promote PTX resistance to multiple tumors. IL-22 enhanced the paclitaxel resistance of lung adenocarcinoma cells by promoting the expression of AKT and Bcl-2 ${ }^{[9]}$. Calpain2 promotes NSCLC progression and contributes to the paclitaxel resistance by activating EGFR/pAKT pathway ${ }^{[10]}$. Inhibition of AKT by specific PI3K-AKT inhibitors (Wortmannin, and LY294002) synergistically increased the efficacy of the paclitaxel-induced apoptosis in ovarian cancer ${ }^{[11]}$. About $15.7 \%$ of AKT1 amplification is found in $\mathrm{ESCC}^{[12]}$, and a recent study has demonstrated that the AKT signaling pathway plays an important role in ESCC metastasis ${ }^{[13]}$. Xanthohumol significantly inhibits AKT kinase activity in an ATP-competitive manner and decreases tumor volume and weight in patient-derived xenografts (PDXs) that highly express AKT. However, xanthohumol has no effect on PDXs that exhibit low expression of AKT in $v^{2}{ }^{[14]}{ }^{[14}$ AKT is also involved in cellular polyploidization, overexpression of AKT restored polyploidy in death effector domain-containing protein (DEDD) deficient mouse decidual cells ${ }^{[15]}$. Yap strongly induce acetyltransferase p300 mediated E3 ligase acetylation through AKT signaling, the Skp2 of acetylation is limited to the cytosol, leading to excessive accumulation of cyclin-dependent kinase inhibitor p27, resulting in the cessation of mitosis and subsequent cellular polyploidy ${ }^{[16]}$.

RhoA, a small GTPase, plays a variety of functions in regulating cell and developmental events, including cytokinesis, cell migration and phagocytosis ${ }^{[17]}$. Cytokinesis is the last step of cell division, which divides heredity and cytoplasmic content into daughter cells. Cytokinesis failure can lead to polyploidy, genetic instability and cancer. Low level of local RhoA activation prevented the accumulation of actin in cleavage furrow and participated in the polyploidization of megakaryocytes $(\mathrm{MK})^{[18]}$.

microRNAs (miRNAs) play an important role in tumor chemotherapeutic resistance and tumor progression ${ }^{[19-21]}$, which also can be regulate PCCs formation by their target genes. In the present study, we induced PCCs by PTX and the miRNA expression profiles were analyzed compared with those of normal cultured cancer cells. 
We found that miR-378d expression was significantly downregulated in PTX induced PCCs. However, miR-378d participation PCCs formation and the impact on the biological behavior of ESCC remains unclear.

\section{Material and methods}

\subsection{Cell culture}

Four human ESCC cell lines (KYSE-30, KYSE-150, KYSE-510, and TE-1) were obtained from the German Resource Center for Biological Material (DSMZ), and 293T cells were purchased from the American Type Culture Collection (USA). The ESCC cell lines were cultured in RPMI1640 (293T cells in DMEM) supplemented with 10\% fetal bovine serum (FBS; (\#04-001-1ACS, BI), $100 \mathrm{units} / \mathrm{mL}$ penicillin, and $100 \mu \mathrm{g} / \mathrm{mL}$ streptomycin and maintained at $37{ }^{\circ} \mathrm{C}, 5 \% \mathrm{CO}_{2}$.

\subsection{ESCC organization source}

A cohort of 610 subjects with ulcerative ESCCs was recruited between 2008 and 2014 from the Department of Thoracic Surgery, the Affiliated Hospital of Jining Medical University (Shandong, PR China). We collected relevant clinical data and prognostic information of patients. Among them, 470 cases were male and 140 cases were female (3.4:1), with ages ranging within $34-83$ years old (mean age $=61$ years). A total of 318 patients had long-term follow-up results, and the mean survival time was 29 months (1-95.2 months).

All biopsies were immediately fixed in $4 \%$ buffered paraformaldehyde, routinely processed, and embedded with paraffin. Tumors were classified according to standard TNM staging guidelines of UICC (TNM Classification of Malignant Tumours, Eighth edition). The study protocol had been reviewed and approved by the local ethics committee. All patients gave written consent for their tissue samples. This research was approved by the ethics committee of Jining Medical University. Each patient signed an informed consent form.

\subsection{Tissue microarray}

Representative areas of the ESCC were marked on each hematoxylin-eosin (H\&E) slide and tissue paraffin block, and the marked areas of tissue paraffin blocks were sampled for TMAs. TMAs were assembled with a tissue-arraying instrument (Beecher Instruments, Silver Springs, MD, USA) as described by Kallioniemi et al. ${ }^{[22]}$.

\subsection{In Situ Hybridization}

ESCC TMA was dewaxed in xylene, rehydrated in alcohol gradient, and washed 
two times with DEPC-PBS. The sections were treated with $2 \mu \mathrm{g} / \mathrm{mL}$ proteinase $\mathrm{K}$ (Roche) for $15 \mathrm{~min}$ at $37^{\circ} \mathrm{C}$ and washed three times with DEPC-PBS. Then, the sections were acetylated $15 \mathrm{~min}$ at room temperature (acetic anhydride in DEPC-water, $6 \mathrm{~N} \mathrm{HCl}$, and triethanolamine) and subsequently washed three times with DEPC-PBS. Sections were prehybridized in hybridization buffer $(50 \%$ formamide; $5 \times$ saline sodium citrate; $\mathrm{pH} 7.0 ; 100 \mu \mathrm{g} / \mathrm{mL}$ sheared salmon sperm DNA, $0.5 \mathrm{mg} / \mathrm{mL}$ yeast tRNA, and $1 \times$ Denhardt's solution) at $58{ }^{\circ} \mathrm{C}$ for $1 \mathrm{~h}$ before the buffer was replaced with hybridization solution containing miR probe. The miR-378d detection probes labeled with digoxin at 5'-end was from Boster (\#MK10502). Probes were diluted in pre-hybridization buffer to a concentration of $5 \mathrm{nM}$ and hybridized with the sections overnight at $58{ }^{\circ} \mathrm{C}$ according to the RNA melting temperature of probes. After hybridization, the sections were washed three times with $2 \times \mathrm{SSC}$ and $0.2 \times \mathrm{SSC}$, permeabilized for immunostaining with $0.1 \%$ Triton X-100, and washed two times with PBS. Unspecific background was blocked with 5\% swine serum diluted in PBS/BSA for $30 \mathrm{~min}$.

\subsection{PTX treatment}

All cell lines were cultured in complete medium until the cells reached $90 \%$ confluence. Different concentrations of PTX were added to the different cells, which were then treated for $24 \mathrm{~h}$. PTX was then withdrawn, the medium was replaced, and the cells were cultured until no significant cell death was observed.

\subsection{Sequencing of miRNA and microarray analysis}

The small RNA of TE-1 control and TE-1-PTX (9 days) treated with PTX was used for miRNA sequencing. The miRNA-sequencing libraries were constructed according to the protocol for the Illumina small RNA sample preparation kit. Sequencing was performed on an Illumina HiSeq 2000 sequencer. Library construction and sequencing were performed at Genergy Biotech (Shanghai). miRNA expression was analyzed with miRdeep 2.0.0.7 ${ }^{[23]}$, and differentially expressed miRNAs were identified using an FDR cutoff value of 0.05. mRNA expression profiling was conducted with Roche NimbleGen Human $12 \times 135$ K Gene Expression Array by KangChen Bio-tech. Raw data were processed with RMA algorithm, and differential expression analysis was performed with $\mathrm{R}$ package limma37 (Version 3.22.7).

\subsection{Cell transfection}

Transfection of plasmids was performed using Lipofectamine ${ }^{\mathrm{TM}} 3000$ reagent (Invitrogen, USA) according to the manufacturer's instructions. Transfection of miRNA mimics or inhibitors (Ribobio, China) was performed using Lipofectamine 
RNAiMAX (Invitrogen, USA) at a final concentration of $20 \mathrm{nM}$.

\subsection{Lentivirus packaging and transduction}

Vectors were packaged in 293FT cells using ViraPower Mix (Genepharma). After culturing for $48 \mathrm{~h}$, lentiviral particles in the supernatant were harvested and filtered by centrifugation at $500 \mathrm{~g}$ for $10 \mathrm{~min}$, and transfected into ESCC cells. The cells were then cultured under puromycin $(10 \mu \mathrm{g} / \mathrm{mL})$ selection for 2 weeks, after which real-time PCR was used to determine the level of miR-378d. Cell lines stably expressing miR-378dinhibitor or negative control (NC) vector were designated as Lv-miR-378d-inhibitor and Lv-miR-NC cells, respectively.

\subsection{Western Blot}

Cells were lysed in ice-cold RIPA buffer containing a protease-inhibitor cocktail (Roche). Protein content was quantified with a BCA protein assay kit (Thermo Fisher Scientific). About $30 \mu \mathrm{g}$ of protein was subjected to electrophoresis, transferred onto PVDF membranes (Millipore), and blocked with 5\% nonfat dry milk in Tris-buffered saline containing $0.1 \%$ Tween 20. Membranes were incubated overnight with the following primary antibodies: anti-AKT (dilution 1:1000; CST), anti-AKT1 (1:1000; CST), anti-p-AKT(Ser473) (1:1000; CST), anti-p-AKT (Thr308) (1:1000; CST), anti$\beta$-catenin (1:2000; Proteintech), anti-ALDH1A1 (1:1000; Abcam), anti-Vimentin (1:500; CST), anti-RhoA antibody (1:500; Proteintech), anti-PARP antibody (1:1000; CST), anti-GAPDH antibody (1:3000; Proteintech) and anti- $\alpha$-tubulin antibody (1:3000; Proteintech) served as endogenous controls. The specific bands were visualized using secondary anti-rabbit or anti-mouse antibody (1:3000; Proteintech), enhanced chemiluminescence detection kit (Millipore), and FluorChem FC2 Multi-Imager II (Alpha Innotech).

\subsection{Transwell migration and invasion assay}

In vitro cell migration assay was performed using transwell chambers $(8 \mathrm{~mm}$ pore size; Corning). Cells were plated in serum-free medium $\left(2 \times 10^{4}\right.$ cells per transwell). Medium containing $15 \%$ FBS in the lower chamber served as a chemoattractant. After $48 \mathrm{~h}$, the nonmigrating cells were removed from the top face of the filters by using cotton swabs, and the migratory cells located on the bottom side of the chamber were stained with crystal violet, air dried, photographed, and counted. Images of five random fields at $10 \times$ magnification were captured from each membrane, and the number of migratory cells was counted. Similar inserts coated with Matrigel were used to determine the cellular invasive potential in the invasion assay. 


\subsection{Matrigel $3 D$ cell culture}

Cells $\left(5 \times 10^{3} / 50 \mu \mathrm{L}\right)$ were seeded onto 96 -well plates with a round-bottom lid made of ultralow attachment polystyrene (\#7007, Costar, USA). The cells were cultured overnight and found to form one sphere per well. After discarding the medium and adding $75 \mu \mathrm{L}$ of melted Matrigel (BD, USA) to resuspend the cell sphere, the mixture was incubated for $30 \mathrm{~min}$ for settling. Finally, $200 \mu \mathrm{L}$ full medium/well was added, and the medium was changed every other day.

\subsection{2. $3 D$ culture scaffolds}

For 3D cultures, the commercial 200- $\mu$ m-thick scaffolds (Alvetex ${ }^{\circledR}$, ReproCELL, Durham, UK) were used and manipulated according to the manufacturer's instructions. To render the scaffold hydrophilic, inserts were first submerged in $70 \%$ ethanol for 1 min, then washed twice with sterile PBS and once with 10\% FBS RPMI-1640 complete medium. In each experiment, $2 \times 10^{6}$ cells were seeded in either Alvetex ${ }^{\circledR}$ scaffold placed in a 12-well plate (covered by $3.5 \mathrm{ml}$ of complete medium). Cultured for 7 days.

\subsection{Colony-formation assays}

Cells were seeded onto six-well plates $\left(5 \times 10^{2}\right.$ cells per plate) and cultured for 10 days. The colonies were stained with $1 \%$ crystal violet for $30 \mathrm{~s}$ after fixation with $10 \%$ formaldehyde for $15 \mathrm{~min}$ and then imaged using the camera of an iPhone 5S (Apple, Inc., Cupertino, CA, USA).

\subsection{Dual-luciferase reporter assay}

In a typical procedure, $293 \mathrm{~T}$ cells $\left(3 \times 10^{4}\right.$ cells per well) grown on a 24 -well plate were co-transfected with luciferase reporter miRGLO-AKT1-3'UTR plasmid (WT or mutation type; Genepharma, Shanghai, China) (200 ng per well) and miR-378d (20 nM) using Lipofectamine ${ }^{\mathrm{TM}} 3000$ (Invitrogen, USA). About $24 \mathrm{~h}$ later, a dualluciferase reporter assay kit (Promega, USA) was used to measure the luciferase and renilla activity of these samples according to the manufacturer's instructions.

\subsection{F-actin cytoskeleton fluorescence staining}

Cells were grown on laminin-coated glass cover slips, fixed in $4 \%$ paraformaldehyde, and stained with Phalloidin (Molecular Probes, USA). Cells were observed using a fluorescence microscope (Leica, Germany).

\subsection{Cell-Viability Assay}

CCK8 was used to assess cell viability. KYSE510 and TE-1 cells $\left(1 \times 10^{4}\right)$ were seeded onto a 96-well plate in quintuplicate per well. About $12 \mathrm{~h}$ later, the cells were incubated with a gradient concentration of therapeutic drugs for $48 \mathrm{~h}$. The medium was 
removed, RPMI1640 (90 $\mu \mathrm{L})$ and CCK8 $(10 \mu \mathrm{L})$ were subsequently added to each well, and the mixture was incubated for $3 \mathrm{~h}$ at $37^{\circ} \mathrm{C}$. A microplate reader was used to measure the optical density (OD) at $450 \mathrm{~nm}$. The degree of drug response for tumor cells was estimated by dividing the half-maximal inhibitory concentration (IC50).

\subsection{Immunohistochemistry (IHC) and Immunofluorescence (IF)}

IHC analysis was performed on the cell-block sections from the cultured cells by using the following primary antibodies: anti-CD133 antibody (1:100; Proteintech), and anti-CD44 (1:100, Proteintech), anti-c-MYC (1:100; Proteintech), anti-Ki-67 (MXB, Fuzhou, China).

Immunofluorescence and confocal microscopy were performed as our previously reported ${ }^{[24,25]}$. Images were captured by using a Zeiss confocal microscope.

\subsection{CD133 ${ }^{+}$cells isolation}

CD133 MicroBead Kit (Miltenyi Biotec) was used for cell sorting. A total of $1 \times$ $10^{7}$ cells was harvested and suspended in $60 \mu \mathrm{L}$ of buffer prior to the addition of $20 \mu \mathrm{L}$ FcR blocking reagent and $20 \mu \mathrm{L}$ CD133 MicroBeads, and the mixture was incubated for 10 minutes at $4^{\circ} \mathrm{C}$. The cells were washed twice with buffer and then suspended in $500 \mu \mathrm{L}$ of buffer. Next, magnetic separation was performed according to the manufacturer's instructions. Unlabeled cells passed through, while labeled cells were retained in the column. Labeled and unlabeled cells were separately collected for further experiments.

\subsection{Sphere-formation assay}

Sphere formation assay was performed as our previously reported ${ }^{[25,26]}$. The KYSE-150 and TE-1 cells were seeded in low-attachment six-well culture plates (Corning, NY, USA) at a density of $1 \times 10^{4}$ cells per well under serum-free conditions consisting of DMEM/F-12 (Life Technologies), $20 \mathrm{ng} / \mathrm{mL}$ epidermal growth factor (Invitrogen), $20 \mathrm{ng} / \mathrm{mL}$ basic fibroblast growth factor (Invitrogen), and $20 \mu \mathrm{L} / \mathrm{mL} \mathrm{B} 27$ (Life Technologies). Images were captured under a microscope after 14 days, and the numbers of spheres (diameter $\geq 100 \mu \mathrm{m}$ ) in all wells were counted.

\subsection{Liver transplantation}

The animal protocol was approved by the ethical review committee of the Affiliated Hospital of Jining Medical University. Eight-week-old male BALB/c nude mice (Beijing Weitong Lihua Laboratory Animal Technology Co., Ltd.) were anesthetized with $4 \%$ chloraldehyde hydrate $(100 \mu \mathrm{L} / 10 \mathrm{~g})$, and body temperature was maintained by heating blankets. The thoracoabdominal skin of nude mice was sterilized 
with $75 \%$ alcohol and iodophor, respectively. After cutting open the skin at the lower right of the cartilago to expose the lobe of the liver, $50 \mu \mathrm{L}$ cell suspension $(25 \mu \mathrm{L}$ serumfree and $25 \mu \mathrm{L}$ of Matrigel containing $5 \times 10^{5}$ cells) was injected into the liver capsule slowly. Then, the syringe was pulled out and the injection port was pressed for 2-5 min with an iodophor cotton ball. Finally, the incision was sutured layer by layer according to the anatomical structure. Mice were sacrificed 44 days after tumor-cell inoculation. Afterwards, liver tissues, lung tissues, and abdominal-metastasis tumors were fixed in $4 \%$ saline-buffered formalin, embedded in paraffin, sectioned at $4 \mu \mathrm{m}$, and stained with H\&E and IHC.

\subsection{Statistical Analysis}

Statistical analyses were performed using the SPSS 13.0 software package (SPSS, Chicago, IL, USA) and GraphPad Prism Software (version 6, La Jolla, CA, USA). For statistical comparison of two groups, two-sided Student's t test with the same variances was used. Differences between variables were analyzed by two-tailed or Fisher exact tests. Survival curves were plotted using the Kaplan-Meier method and compared with log-rank tests. Multivariate survival analysis was performed for all parameters found to be significant in univariate analysis using a Cox regression model. Comparisons between groups for statistical significance were performed with a two-tailed Student $t$ test. Data are presented as the mean $\pm \mathrm{SD}$. P values $<0.05$ were considered significant.

\section{Results:}

\section{1. miR-378d participates in the formation of polyploid cancer cells of ESCC in vitro} and in vivo

PTX is the first-line drug for ESCC, but drug resistance remains a problem. In this study, we used PTX to treat TE-1 cells. From days 2 to 9, the cells died continuously, and only a small number of large cells remained at day 9 . These cells slowly proliferated until day 20. Studies have shown that paclitaxel treatment of tumor cells leads to PGCCs production, then the remaining PTX-treated cells at day 9 (TE-1-PTX) and DMSO-treated cells (TE-1-NC) (Figure 1A) were used to perform DI analysis (Figures 1B, 1C\&1D) and detect miRNA differential expression by sequencing (Figure 1E). The DI analysis results showed that large cells were significant enrichment in the TE-1-PTX, the volume of cells and nuclei increased significantly after PTX treatment (Figure 1B), the large cells were significant enrichment even in the DI $>4(8 \mathrm{~N})$ (Figure 1C), and 
analysis data showed that the ratio of cells DI $>2.5(5 \mathrm{~N})$ were significant enrichment from $6 \%$ to $50 \%$ (Figure $1 \mathrm{D})$.

The remaining large cells and control cells were also used to detect miRNA differential expression by sequencing. miRNA gene-expression profile data showed that miR-378d was significantly downregulated in remaining large cells (Figure 1E). This result suggests that miR-378d play an important role in the formation of large cells. miR-378d has two different chromosomal in humans which named MIR378D1 and MIR378D2, the mature miRNA sequence is the same, and the CCLE data showed that MIR378D1 was not expressed in all cell lines (Figure S1A), and MIR378D2 was expressed in most cell lines and ranked the second highest in oesophageal cell lines (Figure S1B, showed that the miR-378d is MIR378D2. The Table1dada also showed the MIR378D2 expression levels in KYSE-30, KYSE-150,TE-1 and KYSE-510 cells.

Then lentiviral was used to infect stable expression miR-378d-inhibitor in ESCC cell lines, which was similar to PTX treatment that significantly reduced miR-378d expression. The DI analysis results showed that inhibiting miR-378d expression promoted polyploid tumor cells $(\geq 5 \mathrm{~N}$ ) formation both in KYSE-150 and TE-1 cells (Figure 1H), but the increased ratio of polyploid cells was not as significant as the PTX treatment. We detected the mitotic status of cells by immunofluorescence used the antipercentrin antibody and $\alpha$-tubulin antibody, and the most control cells showed normal polarization, but miR-378d suppression showed more multipolarization, indicating polarization disorder (Figure 1G), which may be the cause of polyploidy.

Orthotopic liver transplantation tumor model of nude mice was constructed in our experiments. Both the liver tumors (Figure 1Ha) and abdominial metastatic tumors (Figure $1 \mathrm{Hb}$ ) emerged many large cells and significant heterogeneity after miR-378d silencing. Inhibiting miR-378d also leaded to invasive growth (Figure 1Hb), and the proliferating marker Ki-67 high expression (Figure 1Hd). The data indicated that miR$378 \mathrm{~d}$ inhibition may promote tumor heterogeneity by promoting tumor polyploidy, not just produced polyploid like PTX. 


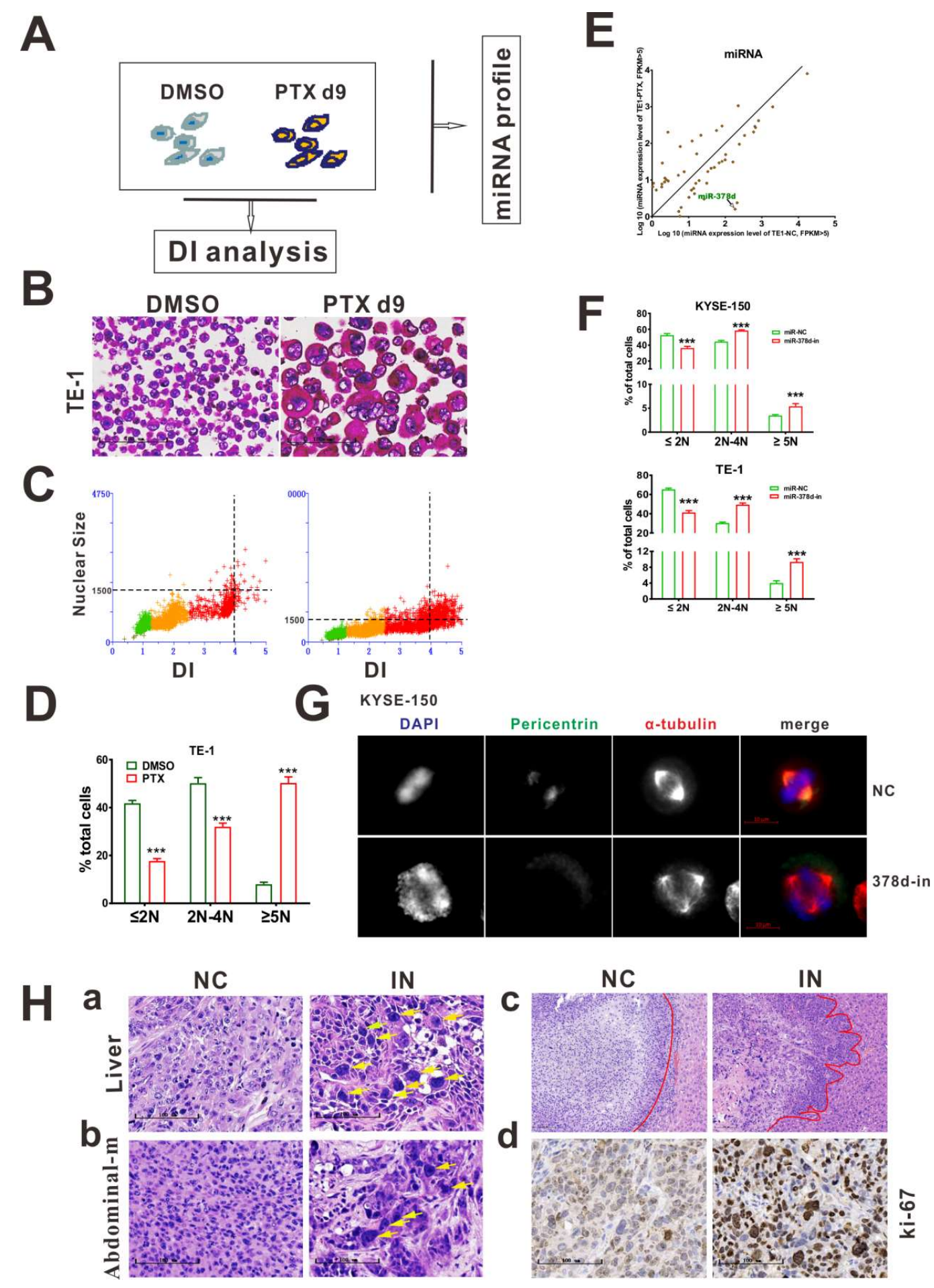

Figure 1. miR-378d participates in the formation of PCCs of ESCC cells. (A) TE-1 cells were treated with DMSO and high concentration of PTX (300 nM) for $24 \mathrm{~h}$ then removed DMSO and PTX and continued to culture for 9 days, the cells were harvested for DI analysis and miRNA profile. (B) Feulgen and Eosin staining for DMSO and PTX 
treated (day 9) TE-1 cells, the volume of cells and nuclei increased significantly after PTX treatment, scale bar $=100 \mu \mathrm{m}$. (C) DNA Index analysis of TE-1 cells after DMSO and PTX treatment. (D) Cell ratio of different DI of total cells for DMSO and PTX treatment cells, repeat the assay three times, $* * * P<0.001$. (E) Scatterplot showing differential mi-RNA expression between TE-1 Ctrl and TE-1 PTX (9 days), FPKM > 5, miR-378d expression level was significantly reduction in PTX treated cells. (F) Cell ratio of different DI of total cells for miR-NC and miR-378d-in cells of KYSE-150 and TE-1, repeat the assay three times, ${ }^{* * *} P<0.001$. (G) Immunofluorescence staining for DAPI, Pericentrin, $\alpha$-tubulin to show cell polarity of miR-NC and miR-378d-in cells of KYSE-150. (H) Orthotopic liver transplantation tumor model of nude mice were used KYSE-150-miR-NC and KYSE-150-miR-378d-in cells respectively, a,b,c Hematoxylin \& Eosin staining for liver tumors and abdominial metastatic tumors, Yellow arrows: large nuclei, Red lines: tumor margins.scale bar $=100 \mu \mathrm{m}$. d, immunohistochemical staining of Ki-67 for abdominial metastatic tumors. scale bar $=$ $100 \mu \mathrm{m}$.

Table 1. The CCLE data of miR-378d expression in 27 oesophagus cells from

\begin{tabular}{l|l}
\hline Ce11 1ines & \multicolumn{1}{|c}{ MIR378D2 Relative Expression } \\
\hline C0L0680N_OESOPHAGUS & 0.390583714 \\
\hline TE5_0ESOPHAGUS & 0.282713611 \\
\hline TE8_0ESOPHAGUS & -0.093942041 \\
\hline KYSE450_OESOPHAGUS & -0.531539773 \\
\hline TE10_0ESOPHAGUS & -0.636216807 \\
\hline ECG110_0ESOPHAGUS & -0.857400541 \\
\hline KYSE30_OESOPHAGUS & -1.11074848 \\
\hline JHES0AD1_OESOPHAGUS & -1.226255504 \\
\hline KYSE150_OESOPHAGUS & -1.23089664 \\
\hline KYSE140_OESOPHAGUS & -1.562493894 \\
\hline KYSE180_OESOPHAGUS & -1.775078164 \\
\hline TE1_OESOPHAGUS & -1.777436638 \\
\hline OE33_0ESOPHAGUS & -1.885956348 \\
\hline KYSE70_OESOPHAGUS & -2.278571477 \\
\hline TE6_0ESOPHAGUS & -2.338848534 \\
\hline KYSE510_OESOPHAGUS & -2.40622878 \\
\hline TE15_0ESOPHAGUS & -2.517624569 \\
\hline
\end{tabular}




\begin{tabular}{l|l}
\hline KYSE270_0ESOPHAGUS & -2.685948849 \\
\hline OE19_0ESOPHAGUS & -2.731908661 \\
\hline TT_0ES0PHAGUS & -2.772512877 \\
\hline TE9_0ESOPHAGUS & -3.156623479 \\
\hline KYSE410_0ESOPHAGUS & -4.02373158 \\
\hline KYSE520_0ESOPHAGUS & -4.559929251 \\
\hline TE11_0ESOPHAGUS & -4.88919334 \\
\hline TE4_0ESOPHAGUS & -4.990699297 \\
\hline 0E21_0ESOPHAGUS & -5.833920453 \\
\hline TE14_0ESOPHAGUS & -13 \\
\hline ES026_0ESOPHAGUS & none \\
\hline ES051_0ESOPHAGUS & none \\
\hline FL01_0ES0PHAGUS & none \\
\hline HCE4_0ES0PHAGUS & none \\
\hline KYAE1_0ESOPHAGUS & none \\
\hline KYSE220_0ESOPHAGUS & none \\
\hline KYSE50_0ESOPHAGUS & none \\
\hline 0ACM51_0ESOPHAGUS & none \\
\hline 0ACP4C_0ESOPHAGUS & none \\
\hline SKGT4_0ESOPHAGUS & none \\
\hline TE12_0ESOPHAGUS & none \\
\hline &
\end{tabular}

\section{2. miR-378d loss expression promotes malignant phenotype of ESCC cells}

Polyploidy and heterogeneity can promote malignant phenotype of tumor cells. Cisplatin and 5-Fu are also first-line clinical chemotherapy drugs. Our data revealed that miR-378d silencing enhanced the resistance of cisplatin at 5, 10, 20, 30, and $40 \mu \mathrm{M}$ for TE-1 and KYSE-510 cells and significantly increased the LC50 of these cells (5-40 and 15-25 $\mu \mathrm{M}$, respectively) (Figure 2A). Consistent with cisplatin treatment, miR$378 \mathrm{~d}$ silencing also promoted 5-Fu resistance at 2.5, 5, 10, 20,10 $\mu \mathrm{g} / \mathrm{mL}$ and increased the LC50 for TE-1 (25 to $40 \mu \mathrm{g} / \mathrm{mL}$ ) and KYSE-510 (5 to $15 \mu \mathrm{g} / \mathrm{mL}$ ) (Figure 2B). miR$378 \mathrm{~d}$ silencing also promoted the monoclonal formation of TE-1 $(P=0.0004)$ and KYSE-510 ( $P=0.0003)$ cells compared with that of control cells (Figure 2C), and promoted microfilaments skeleton formation and extension both in TE-1 and KYSE510 cells (Figure S2A), which indicated epithelial-mesenchymal transition (EMT) occurrence. Transient transfection of miR-378d-mimics inhibited the migration 
$(P<0.001)$ and invasion $(P<0.001)$ ability of TE-1 cells (Figure S2B), while miR-378d inhibition promoted the migration $(P<0.001)$ (Figure S2Cc) and invasion $(P<0.001)$ (Figure S2Cb) ability of TE-1 and KYSE-510 cells. KYSE-150 cells were easy to form spheres in suspension state within $24 \mathrm{~h}$, but miR-37d inhibition promoted the spheres dissemination after $48 \mathrm{~h}$, while control group was still spheres (Figure S2D). The spheres were transferred into the Matrigel and cultured for 8 days, miR-378d inhibition showed invasive growth (Figure 2D). The invasive ability was further detected by 3D culture on $3 \mathrm{D}$ Alvetex ${ }^{\circledR}$, a highly loose crosslinked polystyrene scaffold, and the data showed that miR-378d inhibition promoting cell invasion in 3D scaffold (Figure 2E).

Studies have shown that PCCs and its daughter cells have cancer stem-like cell characteristics. PTX inducing PCCs were significantly high expression stem markers CD133, CD44 and c-MYC (Figure S3A). CD133 ${ }^{+}$and $\mathrm{CD}^{-333^{-}}$cells were sorted to perform DNA ploidy analysis, significant large cells were enriched in $\mathrm{CD} 133^{+}$cells of TE-1 and KYSE-150 (Figure 2F \& S3B). Compared with CD133- cells, the number of $\mathrm{CD}_{133^{+}}$mitotic figures decreased (Figure $2 \mathrm{~F}$ ). According to the diameter classification, the proportion of $\mathrm{CD}_{133^{+}}$cells were mainly concentrated in small $(<20 \mu \mathrm{m})$ and large (>30 $\mu \mathrm{m}$ ) diameter cells, while the proportion between 20-30 $\mu \mathrm{m}$ decreased compared

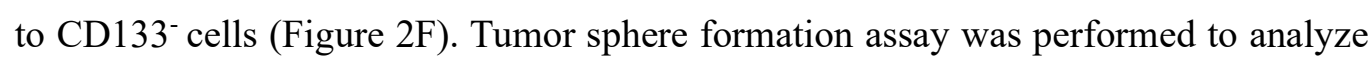
the stem cell capability of KYSE-150 and TE-1 cell after miR-378d inhibition, and the data showed that compared with the control group, miR-378d inhibition significantly promoted sphere formation both in KYSE-150 and TE-1 cells (Figure 2G).

These results suggest that miR-378d inhibition promoted chemo-resistance, monoclonal formation, EMT, migration, invasion and stemness. 
A TE-1
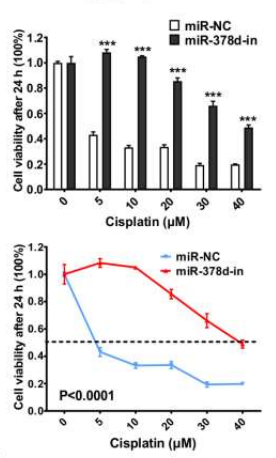

C

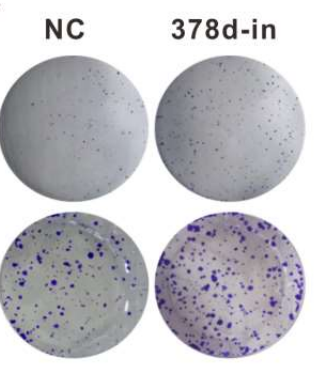

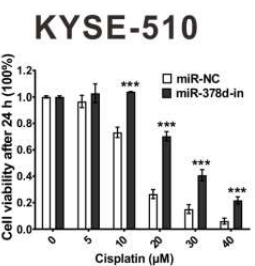
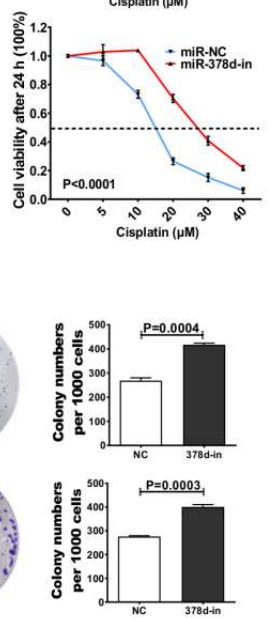

B
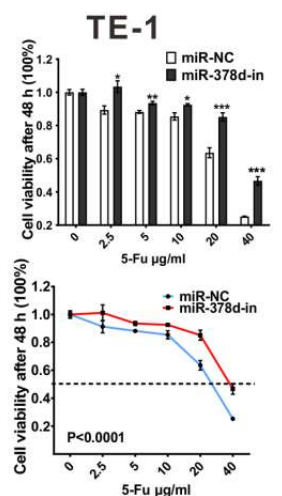

D
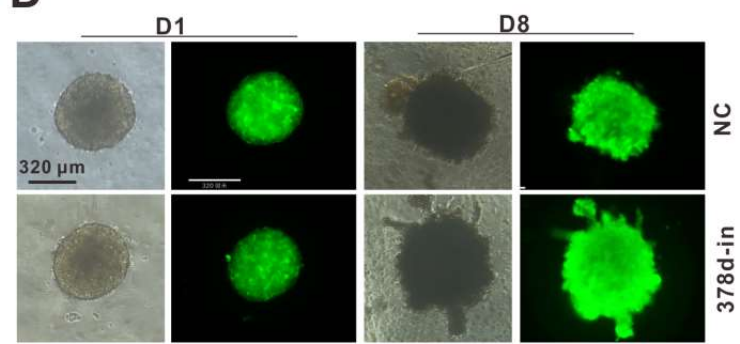

E

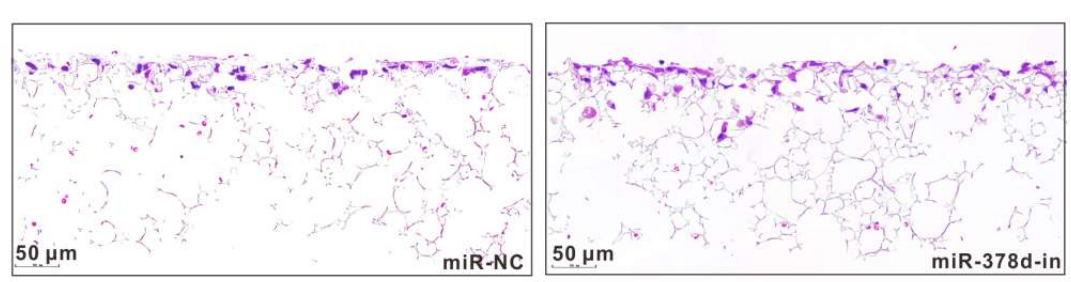

$\mathbf{F}$
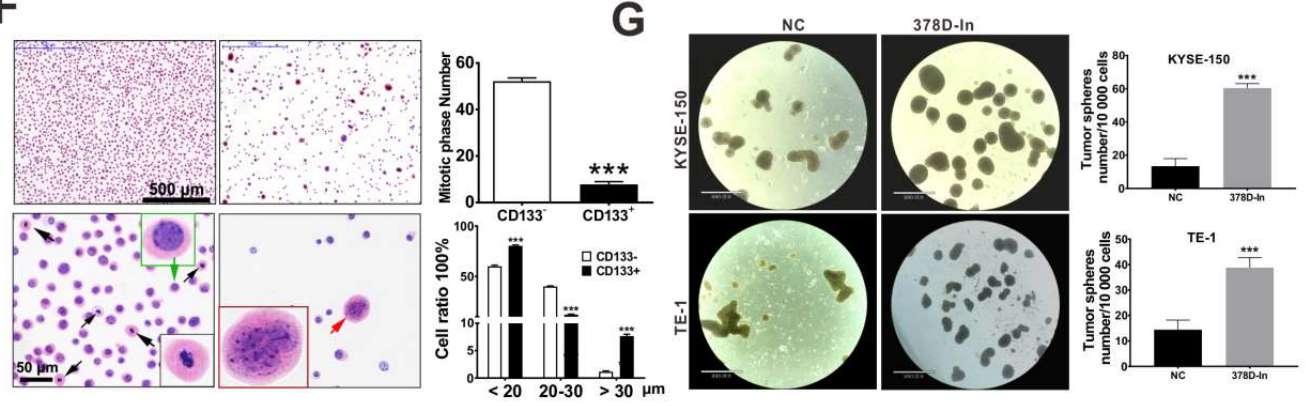

Figure 2. miR-378d loss expression promotes malignant phenotype of ESCC cells. (A-

C) Cell-Viability Assay, the cells were treated with different concentrations of 5- Fu or cisplatin for $24 \mathrm{~h}$, then treated with CCK 8 for $30 \mathrm{~min}$, and detection the OD at $450 \mathrm{~nm}$. $\mathrm{T}$ test and ANOVA test were used for statistical differences analysis, five repeat wells per concentration, $* P<0.05, * * P<0.01, * * * P<0.001$. (C) Silencing of miR-378d promoted the monoclone formation of TE-1 $(P=0.0004)$ and KYSE510 $(P=0.0003)$ cells, repeat each experiment three times. (D) 3D tumor-sphere formation, in Matrigel. (E) HE staining for KYSE-150 cells cultured on 3D Alvetex ${ }^{\circledR}$, a highly loose 
crosslinked polystyrene scaffold, to detect the depth of cell invasion. (F) Feulgen and eosin staining for $\mathrm{CD} 33^{-}$and $\mathrm{CD} 133^{+}$cells of TE-1 sorting by magnetic beads, black arrow: mitotic figures, green arrow: normal size tumor cell, red arrow: polyploid cell. Histogram analysis mitotic cell number and cell ratio of different sizes, ${ }^{* * *} P<0.001$. Scale bars $=500 \mu \mathrm{m}$ or $50 \mu \mathrm{m}$. (G) Tumor sphere formation assays in KYSE-150 and TE-1 cells. The graph is the summarized data of three independent experiments, ${ }^{* * *} P$ $<0.001$. Scale bar $=890 \mu \mathrm{m}$.

\section{3. miR-378d loss expression promotes metastasis in vivo}

Liver is a common metastasis site of ESCC, so liver-transplantation assay was performed to analyze the metastatic ability of tumor cells without miR-378d expression. KYSE-150-miR-NC and KYSE-150-miR-378d-inhibitor cells were transplanted into subcapsular liver of BALB/c nude mice (hereafter denoted as NC-mice and In-mice, respectively). The experiment was terminated after 44 days. The body weight of Inmice was significantly lower than that of NC-mice at day $44(P=0.011$, Figures 3A \& $3 \mathrm{~B})$. Before the end of the experiment, NC-mice did not die (0/6), but two IN-mice (2/6) died at day 35 and 38, respectively (Figure 3C). Although the number of liver nodules in In-mice was more than that in NC-mice, no statistical difference (Figure 3D \& 3E) was observed. Tumor cells basically metastasized to the abdominal cavity, and no metastasis was found in all lung tissues. Abdominal metastatic tumors appeared in 5/6 of the NC-mice and $6 / 6$ of the In-mice (Figure 3F). The number of abdominalmetastasis tumors in In-mice was more than that in NC-mice (Figure 3G, $P=0.026$ ), and the volume was also larger than that of the NC-mice. These data showed that loss of miR-378d expression promoted tumor metastasis. 

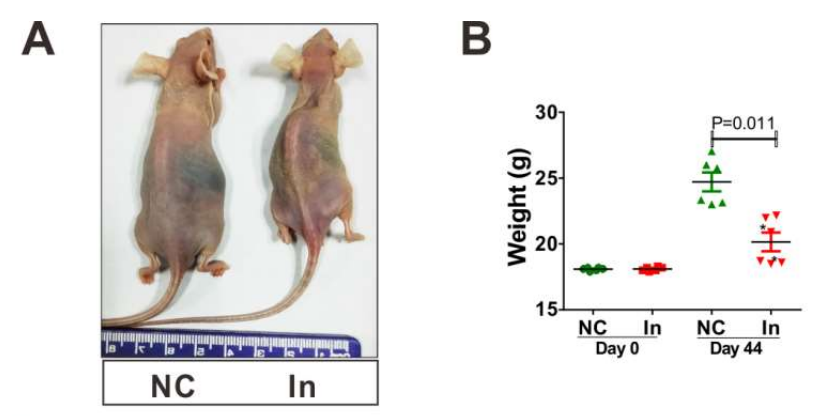

C

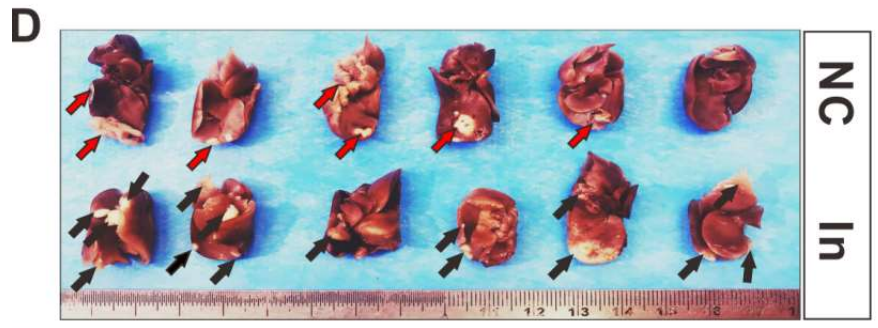

E
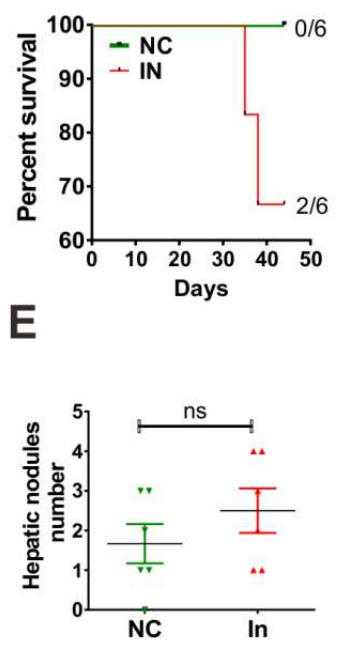

$\mathbf{F}$

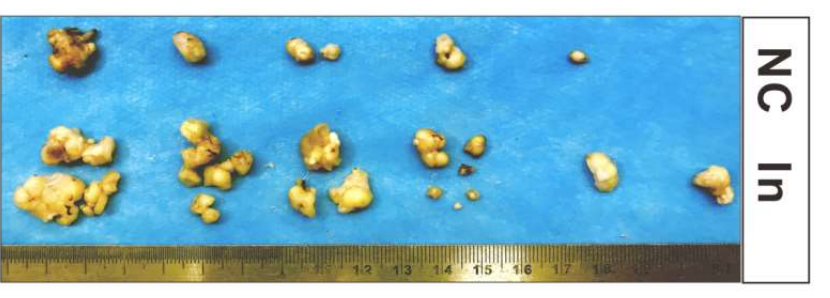

G

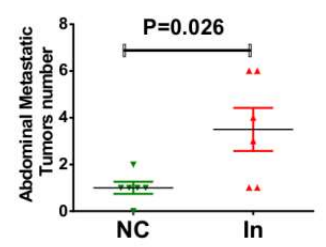

Figure 3. miR-378d inhibition promotes metastasis. (A) Tumor-bearing mice with liver-transplantation tumors, 6six BALB/c nude mice in each group. Red and black arrows represent tumor nodules. (B) Mouse body weight at days 0 and 44, *: mouse died before day 44. Compared with NC-mice, In-mice lost body weight. $P=0.011$. (C) Survival rate of mice. (D) Liver with transplantation tumors. (E) Number of hepatic nodules, ns: no significance. (F) Abdominal metastatic tumors. (G) Number of abdominal-metastasis tumors. $P=0.026$.

\section{4. miR378d regulated AKT and RhoA}

The pathway-enrichment statistical scatterplot showed that TE-1 NC and TE-1PTX differential miRNA target molecules were enriched in the PI3K-AKT signaling pathways (Figure 4A). Differential mRNA molecules were closely related to the AKT signaling pathway. Gene-set enrichment analysis (GSEA) data also showed that differentially expressed genes of TE-1-PTX were enriched in AKT, Tumorigenesis, CTNNB1 oncogenic and RhoA (Figure 4B). miR-378d target genes were predicted by 
online software miR-TarBase (http://mirtarbase.cuhk.edu.cn/php/index.php) and $\begin{array}{lll}\text { TarBase } & \text { V.8 (http://carolina.imis.athena- }\end{array}$ innovation.gr/diana_tools/web/index.php?r=tarbasev8\%2Findex), and the pathwayenrichment statistical scatterplot showed that the miR-378d target genes were enriched in AKT and CTNNB1 signaling pathway (Figure 4C).

The miRTarBase online software predicted that AKT1 was a potential target gene of miR-378d (Figure 4D \& 4E). Dual-luciferase activity assay revealed that miR-378d targeted the 3'UTR of AKT1 mRNA (Figure 4F). Transient transfection of miR-378d mimics to TE-1 cells decreased the AKT1 and p-AKT (T308 and S473) protein expression levels (Figure 4G). Stable transfection of miR-378d inhibitor to TE-1 and KYSE-510 cells increased the total AKT1 and p-AKT (T308 and S473) expression (Figure 4H). The results showed that miR-378d directly regulated AKT1 expression. CTNNB1 ( $\beta$-catenin) oncogenic signature, which was the AKT regulated pathway. The protein expression levels of $\beta$-catenin and the downstream target genes vimentin and ALDH1A1 decreased in cells with miR-378d high expression (Figure 4I), whereas miR-378d absent increased these protein levels (Figure 4J). The EMT marker Ecadherin significantly upregulated in miR-378d high expression TE-1 cells, and downregulated in TE-1 cells with miR-378 inhibition (Figure 4I\&4J). The data showed that miR-378d absence activated the $\beta$-catenin pathway, and promoted the EMT of ESCC cells.

RhoA is a key regulatory protein for cytokinesis, we detected the RhoA expression in miR-378d inhibition ESCC cells, and RhoA expression were all downregulated in TE-1, KYSE-150 and KYSE-510 cells (Figure 4K). The results suggest that miR-378d inhibition may promote polyploid production by reducing RhoA expression. Then we detected the RhoA expression after AKT inhibition by small molecule inhibitor MK2206, and all of the four ESCC cell lines showed RhoA protein reduced expression (Figure 4L). The results suggest that miR-378d may regulate polyploid production and malignant phenotype through AKT and RhoA. 

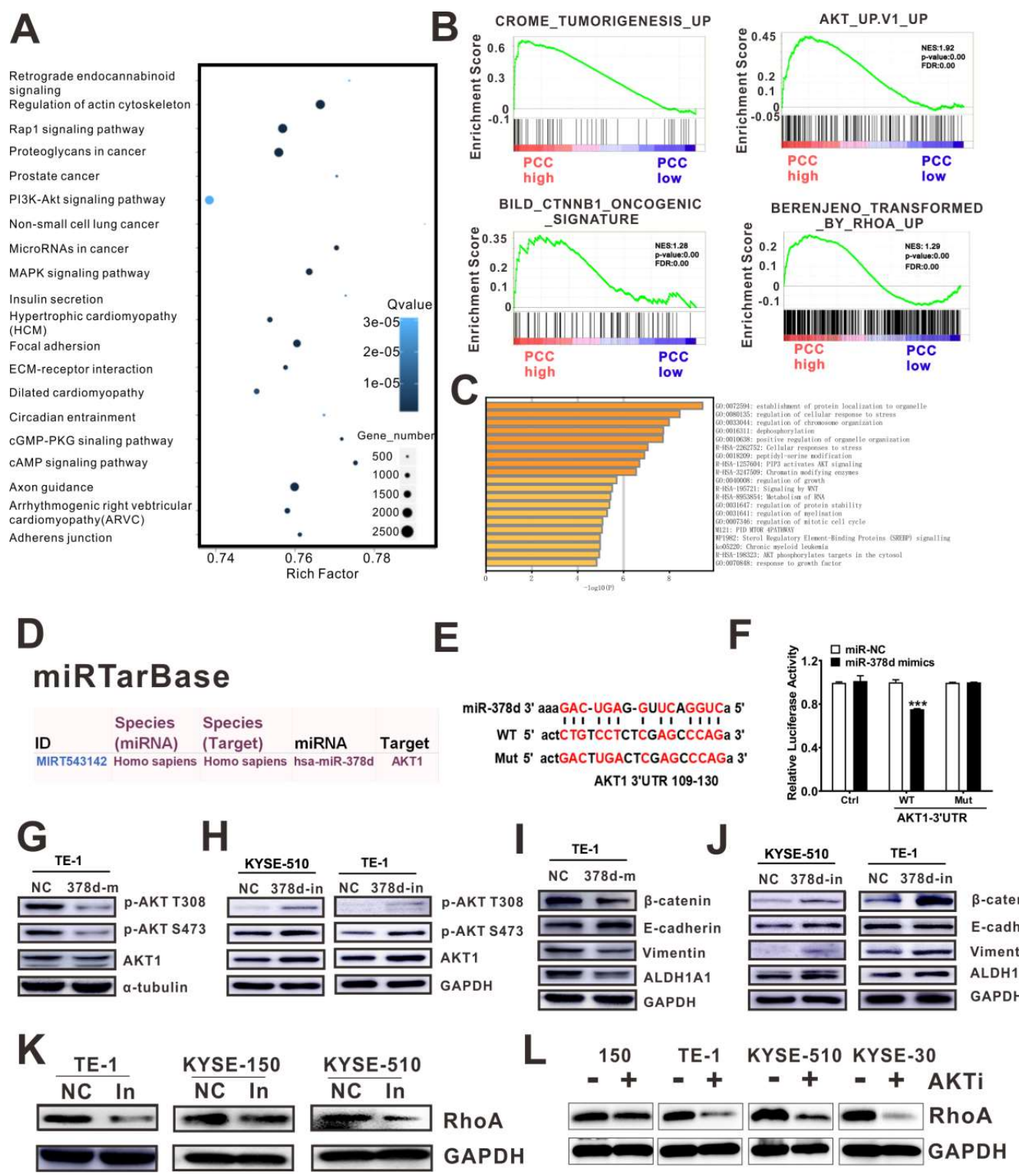

Figure 4. miR378d regulated AKT and RhoA. (A) Differentially expressed miRNA target gene-enrichment analysis. (B) Gene-set enrichment analysis (GSEA) of TE-1 NC and TE-1 polyploid tumor cells for tumorigenesis, AKT signaling, AKT downstream CTNNB1 signaling, and cytokinesis related RhoA signaling. (C) Gene-enrichment analysis of miR-378d predicting target genes. (D) Software prediction of binding sites between miR-378d and 3'UTR of AKT1 mRNA. (E) luciferase reporter vector designed containing wild type or mutation type of AKT1 mRNA 3'UTR (109-130 bp). (F) Duluciferase activity assay; miR-378d reduced the luciferase activity of WT-AKT1-3'UTR but not that of Mut-AKT1-3'UTR. (G) Western blotting detected the protein expression 
levels of p-AKT and AKT1; GAPDH served as an internal reference. (H) Western blotting detected the protein expression levels of p-AKT and AKT1; GAPDH served as an internal reference. (I) Western blotting assay detected the expression levels of $\beta$ catenin, E-cadherin, ALDH1A1, and Vimentin. GAPDH served as an internal reference. (J) Western blotting assay detected the expression levels of $\beta$-catenin, E-cadherin, ALDH1A1, and Vimentin. GAPDH served as an internal reference. Western blotting assay detected the expression levels of RhoA after miR-378d inhibition (K) or MK2206 treatment $(\mathbf{L})$ in ESCC cells.

\section{5. miR-378d regulate polyploidization and deploidization through regulation of RhoA} and $A K T$

To investigate the role of miR-378d, AKT and RhoA in polyploid formation, DNA ploidy analysis for KYSE-150 cells were performed after miR-378d inhibition, AKT inhibition, RhoA inhibition, or combined inhibition of AKT and RhoA in KYSE-150 cells. Compared with control cells, inhibition of miR-378d, AKT, RhoA significantly promoted polyploid production, the effect of combined inhibition of AKT and RhoA on polyploid formation was further strengthened (Figure 5A). The contents of polyploid cells in 25000 cells in each group were counted by software, the results are consistent with Figure 4A that inhibition of miR-378d, AKT, RhoA significantly promoted polyploid production, combined inhibition of AKT and RhoA on polyploid formation was further strengthened (Figure 5B). Suppress AKT significantly suppresses RhoA expression, combination of RhoA and AKT inhibitors works best on RhoA inhibition (Figure 5C).

To determine whether miR-378d regulated the downstream genes ( $\beta$-catenin, vimentin, and ALDH1A1) expression by targeting AKT1, we treated TE-1 cells with inhibitors to suppress AKT or $\beta$-catenin expression. AKT inhibitor MK-2206 downregulated the protein expression levels of AKT1, p-AKT, and the downstream $\beta$ catenin and ALDH1A1 in TE-1-miR-378d-inhibitor cells (Figure 5D), suggesting that miR-378d silencing activated the AKT- $\beta$-catenin signaling pathway. Our data also showed that inhibiting $\beta$-catenin significantly inhibited the expression of its target genes 
(Vimentin and ALDH1A1), and in turn inhibited AKT1 expression, main inhibition of S473 of AKT, but little effect on T308 of AKT (Figure 5D). Thus, AKT and $\beta$-catenin formed a positive feedback loop, which may play an important role in promoting malignant phenotype of tumors.

RhoA protein expression level was also reduced in miR-378d-in cells and in $\beta$ catenin inhibition cells, RhoA expression was almost undetectable though miR-378d inhibition combined AKT inhibition (Figure 5D), which indicated that miR-378d-in downregulated RhoA independent of AKT1, a relatively low RhoA expression level may due to AKT activation.

The possible molecular mechanisms for regulating polyploidization/polyploidization and tumor heterogeneity are that: miR-378d expression was downregulated under stress; miR-378d reducing facilitated polyploid production by inhibiting RhoA expression, the number of polyploid cells maintained at a relatively high level; tumor heterogeneity generated through depolyploidization via AKT signaling activation (Figure 5E). 


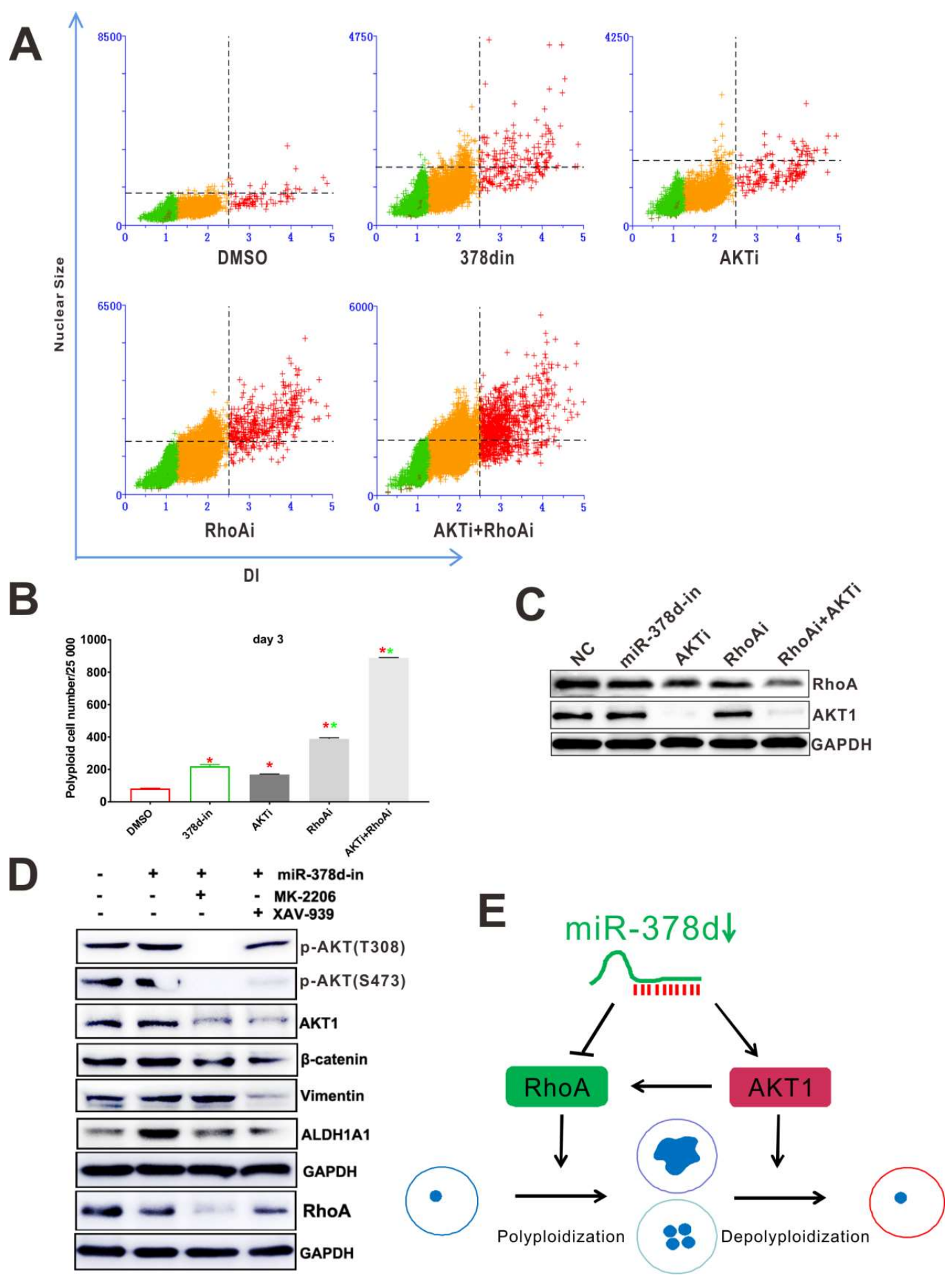

Figure 5. miR-378d regulate polyploidization and deploidization through regulation of RhoA and AKT1. (A) DI analysis for KYSE-150 cells after miR-378d-in, AKT inhibitor MK-2206 $(10 \mu \mathrm{M})$, RhoA inhibitor CCG-1423 (5 $\mu \mathrm{M}$, CCG-1423 is a novel inhibitor of RhoA transcription signaling), and combined inhibitors of AKT and RhoA. (B) Polyploid cancer cells number per 25000 cells. Repeat each experiment three times. 
Red *: Comparison with DMSO groups, $P<0.001$; Green *: Comparison with miR378d-in groups, $P<0.001$. (C) Western blot to detect RhoA, AKT1 and GAPDH after miR-378d-in, MK-2206 (10 $\mu \mathrm{M})$, CCG-1423 $(5 \mu \mathrm{M})$ treatment or combined treatment of MK-2206 and CCG-1423.(D) Western blot to detect p-AKT, total AKT1, $\beta$-catenin, Vimentin, ALDH1A1, RhoA and GAPDH after miR-378d-in, MK-2206 (10 $\mu \mathrm{M})$ or XAV939 $(10 \mu \mathrm{M})$ treatment. (E) Molecular mechanism pattern, miR-378d regulates polyploidization and deploidization through regulation of RhoA and AKT1.

\subsection{Patients with low miR-378d expression had poor prognosis}

We detected miR-378d expression by ISH in a ESCC tissue array containing 610 ESCC samples because the incompleteness of the paraffin section and antigen repair led to tissue shedding. Finally, 596 specimens were obtained. Most ESCC patients (388/596, 65.10\%) showed the miR-378d low expression (Figure 6A), miR-378d positive staining $(208 / 596,34.90 \%)$ was primarily in the cytoplasm of squamous epithelial and rarely expressed in nuclei (Figure 6B). miR-378d expression level had no clinicopathological significance with age $(P=0.269)$, gender $(P=0.864)$, tumor size $(P=0.757)$, differentiation $(P=0.249)$, stage $(P=0.305)$, LNM $(P=0.296)$, nerve invasion $(P=0.141)$, vascular invasion $(P=0.578)$, and invasion depth $(P=0.101)$ of ESCC patients (Table 2). However, our data showed that miR-378d expression was significantly negatively associated with the overall survival rate (Figure $6 \mathrm{C} ; P=0.0009$ ) of ESCC patients.
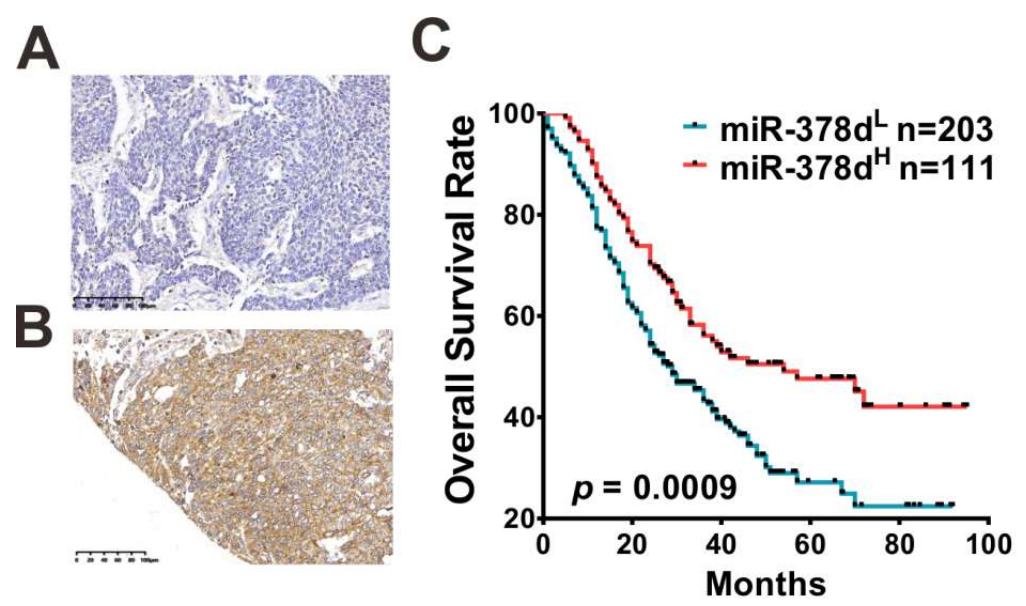
Figure 6. Patients with low miR-378d expression had poor prognosis. miR-378d expression was detected by in situ hybridization using the ESCC TMA, (A) low expression of miR-378d, and (B) high expression of miR-378d. (C) Overall survival rate of ESCC patients according to miR-378d expression levels.

Table 2. miR-378d expression in ESCC patients and its clinicpathological significance, 596 case

\begin{tabular}{|c|c|c|c|c|c|}
\hline $\begin{array}{c}\text { Clinical } \\
\text { information }\end{array}$ & $\begin{array}{c}\text { miR-378d } \mathrm{d}^{\mathrm{Low}} \\
\mathrm{N}(\%)\end{array}$ & $\begin{array}{c}\mathrm{miR}-378 \mathrm{~d}^{\mathrm{High}} \\
\mathrm{N}(\%)\end{array}$ & total & $\chi^{2}$ & $P$ \\
\hline \multicolumn{6}{|l|}{ Age(years) } \\
\hline$\geq 61$ & $198(63.06)$ & $116(36.94)$ & 314 & 1.220 & 0.269 \\
\hline$<61$ & $190(67.38)$ & $92(32.62)$ & 282 & & \\
\hline \multicolumn{6}{|l|}{ Gender } \\
\hline Male & $299(65.28)$ & $159(34.72)$ & 458 & 0.029 & 0.864 \\
\hline Female & $89(64.49)$ & $49(35.08)$ & 138 & & \\
\hline \multicolumn{6}{|c|}{ Tumor size $(\mathrm{cm})$} \\
\hline$>4$ & $143(65.90)$ & $74(34.10)$ & 217 & 0.096 & 0.757 \\
\hline$\leq 4$ & $245(64.64)$ & $134(35.36)$ & 379 & & \\
\hline \multicolumn{6}{|l|}{ Stage } \\
\hline $\mathrm{I}+\mathrm{II}$ & $112(68.29)$ & $52(31.71)$ & 164 & 1.052 & 0.305 \\
\hline III+IV & $275(63.81)$ & $156(36.19)$ & 431 & & \\
\hline \multicolumn{6}{|l|}{ LNM } \\
\hline Negative & $199(63.17)$ & $116(36.83)$ & 315 & 1.091 & 0.296 \\
\hline Positive & $189(67.26)$ & $92(32.74)$ & 281 & & \\
\hline \multicolumn{6}{|l|}{ Nerve invasion } \\
\hline Negative & $343(66.22)$ & $175(33.78)$ & 518 & 2.168 & 0.141 \\
\hline Positive & $45(57.69)$ & $33(42.31)$ & 78 & & \\
\hline \multicolumn{6}{|l|}{$\begin{array}{l}\text { Vascular } \\
\text { invasion }\end{array}$} \\
\hline Negative & $359(64.80)$ & $195(35.20)$ & 554 & 0.310 & 0.578 \\
\hline Positive & $29(69.05)$ & $13(30.95)$ & 42 & & \\
\hline \multicolumn{6}{|l|}{ Differentiation } \\
\hline High & $206(64.58)$ & $113(35.42)$ & 319 & 2.778 & 0.249 \\
\hline Middle & $173(64.79)$ & $94(35.21)$ & 267 & & \\
\hline Low & $9(90.00)$ & $1(10.00)$ & 10 & & \\
\hline \multicolumn{6}{|c|}{ Tumor location } \\
\hline Up & $105(68.18)$ & $49(31.82)$ & 154 & 2.534 & 0.282 \\
\hline Middle & $179(63.03)$ & $105(36.97)$ & 284 & & \\
\hline
\end{tabular}




\begin{tabular}{cccccc} 
Down & $9(81.82)$ & $2(18.18)$ & 11 & & \\
Invasion depth & & & & & \\
Mucous layer & $19(82.61)$ & $4(17.39)$ & 23 & 4.588 & 0.101 \\
Muscle layer & $101(68.24)$ & $47(31.76)$ & 148 & & \\
Whole layer & $267(62.97)$ & $157(37.03)$ & 424 & & \\
\hline
\end{tabular}

\section{Discussion}

Most research has primarily focused on miR-378a and miR-378b ${ }^{[27]}$, only a few studies have been performed on miR-378d and inflammation ${ }^{[28]}$ or infection ${ }^{[29]}$. In the current work, miR-378d significantly decreased in PCCs, however, the role of miR$378 \mathrm{~d}$ in ESCC remains unclear. In this study, we found that miR-378d inhibition promoted polyploid cancer cells formation, heterogenicity, chemo-resistance, monoclonal formation, EMT, migration, invasion, stemness and metastasis of ESCC cells. These results indicate that miR-378d is a tumor suppressor, and actually, miR$378 \mathrm{~d}$ expression is a good prognostic factor for ESCC patients.

In this study, PTX was used to induce polyploid production, PTX is a mitotic inhibitor widely used in the treatment of cancer patients by inhibiting cell division and inducing apoptosis. A low dose $(30 \mathrm{nM})$ of PTX treatment of esophageal cancer cells results in more than $90 \%$ of cells forming PCCs without apoptosis. While high doses of PTX treatment can lead to massive cell death, but a certain percentage of the surviving cells forming PGCCs, a more malignant cell type ${ }^{[6,8,30]}$.

PGCCs exhibit higher plasticity than traditional cancer stem cells and can differentiate into a variety of tissues, including adipose tissue, cartilage, bone and other stromal or fibroblast cells ${ }^{[30]}$. There are two asymmetric cell division patterns in the PGCCs: budding and rupture, which can occur alone or together. Budding usually occurs on PGCCs branches, while PGCCs cells containing multiple nuclei usually rupture, followed by the release of large numbers of small cells ${ }^{[31]}$. CD133 has been suggested as a broad-spectrum marker for cancer stem cells (CSCs). $\mathrm{CD}_{133^{+}}$cells are small, regular and round with small microvilli, in some fields, several giant cancer cells (GCCs) in the $\mathrm{CD} 133^{+}$cell group were identified under the light microscope, most of them were polynuclear cells in NPC cells [25]. In our study, CD133 ${ }^{+}$cells contained more large and small cells than $\mathrm{CD} 133^{-}$cells, the large cells are PGCCs and the small cells maybe 
the daughter cells of PGCCs, and medium size cells are non tumor-stem cells. Therefore, the clinical use of PTX should be re-evaluated.

PGCCs achieve rapid and malignant growth via efficient DNA replication and cell division ${ }^{[32]}$. PGCCs daughter cells promoted lymph node metastasis by expressing EMT related proteins ${ }^{[32]}$. Spectral karyotype (SKY) analysis found that PGCCs derived daughter cells obtained new cancer genome, and new chromosomal recombination, including deletion and translocation occurred in daughter cells ${ }^{[33]}$. These studies suggest that the PGCCs daughter cells have stronger migration and invasion ability than diploid tumor cells.

Our study showed that miR-378d inhibition promoted malignant ability of ESCC cells, but did not significantly increase the proportion of PCCs compared with paclitaxel treatment. Thus, like cancer stem cells, the proportion of PCCs in the cell population is not high, highly efficient generation of daughter cells may be an important reason for PCCs/PGCCs to promote malignant phenotype and tumor heterogeneity.

PI3K/AKT pathway are closely related with tumorigenesis, proliferation, growth, EMT, invasion, metastasis, stem-like phenotype and drug resistance of cancer cells, but PI3K or AKT inhibitors as monotherapy for different cancers have so far been limited $^{[34]}$. In this study, miR-378d directly target regulate AKT1, and miR-378d inhibition activated the AKT- $\beta$-catenin signaling pathway, and promoted the EMT marker vimentin and CSCs marker ALDH1A1 expression, which may promote malignant phenotypes of ESCC cells.

Our data also showed that mi-378d inhibition decreased the RhoA expression. RhoA is a key protein in regulating cytokinesis ${ }^{[35]}$, and cytokinesis is the last step of mitosis, cytokinesis failure causes polyploid formation. miR-378d inhibition may promote PCCs formation by reducing RhoA expression.

AKT or RhoA inhibition alone promoted PCCs formation, and the combination of AKT and RhoA inhibition significantly promoted PCCs production. Then we study the relationship between AKT and RhoA, AKT inhibition by MK2206 decreased the RhoA expression, we also found that $\beta$-catenin inhibition also reduced RhoA expression. Therefore, our results suggest that miR-378d inhibition may promote polyploidization by inhibiting the expression of RhoA and promoting de polyploidization by promoting the activation of AKT and thus promoting the malignant phenotype of tumor.

\section{Conclusions}


miR-378d inhibition promoted PCCs formation, heterogenicity, chemo-resistance, monoclonal formation, EMT, migration, invasion, stemness and metastasis of ESCC cells. miR-378d can target downregulated AKT1, and inactivating the AKT- $\beta$-catenin signaling pathway, miR-378d and AKT can also regulated RhoA expression. AKT and RhoA regulated polyploidization and depolyploidization. Therefore, miR-378d expression is a good prognostic factor of ESCC patients and regulates polyploidization and malignant phenotype of tumor cells through AKT and RhoA.

Supplementary Materials: Figure S1. miR-378D1 and miR-378D2 expression levels in different cells lines, Figure S2. miR-378d inhibits EMT, migration and invasion. Figure S3. PCCs are tumor-like stem cells.

Author contributions: WW designed the study. LL, RYZ, RZ, YRL, and YKL oversaw the study. HXW, HXS, XF, and SQW provided clinical samples. JP, SSS, JY, ZJL, JLL, ZZY, HYZ and DLG performed the majority of the experiments and analyzed the data. JP, JJL, and SJH performed a number of the animal experiments. LL provided research assistance. RYZ, YRL, YKL, and RYZ reviewed and edited the article. WW takes responsibility for the accuracy of the data analysis. WW wrote the article.

Funding: This research was supported by The National Natural Science Foundation of China (81802945), Jining Science and Technology Key Research and Development Plan (2018SMNS006), The PhD Research Foundation Affiliated Hospital of Jining Medical University (2016-BS-002); The cultivation project of the National Natural Science Foundation of Jining Medical University (JYP201733, JYP201731); Research support found for young teachers of Jining Medical University (JY2017FS007, JYP201731, JYFC2019FKJ048, JYFC2019FKJ037, JYFC2019FKJ165). Youth Innovation and technology support program in Shandong Province (2020KJL003).

Institutional Review Board Statement: This study was approved by the institutional review board of the Affiliated Hospital of Jining Medical University, Jining, China (2018-FY-040).

Informed Consent Statement: Written informed consent has been obtained from the patient(s).

Data Availability Statement: Data are available upon reasonable request. We may be able to share de-identified participant data with researchers following the publication of this manuscript. Requests for data should be directed to the corresponding author.

Conflict of Interest:The authors declare that they have no conflict of interest.

\section{References}

1. Xiong, J.X.;Wang, Y.S.;Sheng, J.;Xiang, D.;Huang, T.X.;Tan, B.B.;Zeng, C.M.;Li, H.H.;Yang, J.;Meltzer, S.J. et al. Epigenetic alterations of a novel antioxidant gene SLC22A3 predispose susceptible individuals to increased risk of esophageal cancer. Int J Biol Sci 2018, 14, 1658-1668.

2. Abbas, G.; Krasna, M. Overview of esophageal cancer. Ann Cardiothorac Surg 2017, 6, 131-136. 
3. Tomasello, G.; Ghidini, M.; Barni, S.; Passalacqua, R.; Petrelli, F. Overview of different available chemotherapy regimens combined with radiotherapy for the neoadjuvant and definitive treatment of esophageal cancer. Expert Rev Clin Pharmacol 2017, 10, 649-660.

4. Qu, Y.; Zhang, L.; Rong, Z.; He, T.; Zhang, S. Number of glioma polyploid giant cancer cells (PGCCs) associated with vasculogenic mimicry formation and tumor grade in human glioma. $J$ Exp Clin Cancer Res 2013, 32, 75.

5. Fei, F.; Zhang, D.; Yang, Z.; Wang, S.; Wang, X.; Wu, Z.; Wu, Q.; Zhang, S. The number of polyploid giant cancer cells and epithelial-mesenchymal transition-related proteins are associated with invasion and metastasis in human breast cancer. J Exp Clin Cancer Res 2015, 34, 158.

6. Niu, N.; Mercado-Uribe, I.; Liu, J. Dedifferentiation into blastomere-like cancer stem cells via formation of polyploid giant cancer cells. Oncogene 2017, 36, 4887-4900.

7. Mirzayans, R.; Andrais, B.; Murray, D. Roles of Polyploid/Multinucleated Giant Cancer Cells in Metastasis and Disease Relapse Following Anticancer Treatment. Cancers (Basel) 2018, 10,

8. Zhang, S.; Mercado-Uribe, I.; Liu, J. Tumor stroma and differentiated cancer cells can be originated directly from polyploid giant cancer cells induced by paclitaxel. Int J Cancer 2014, 134, 508-18.

9. Huang, Z.; Gao, Y.; Hou, D. Interleukin-22 enhances chemoresistance of lung adenocarcinoma cells to paclitaxel. Hum Cell 2020, 33, 850-858.

10. Xu, F.; Gu, J.; Lu, C.; Mao, W.; Wang, L.; Zhu, Q.; Liu, Z.; Chu, Y.; Liu, R.; Ge, D. Calpain-2 Enhances Non-Small Cell Lung Cancer Progression and Chemoresistance to Paclitaxel via EGFR-pAKT Pathway. Int J Biol Sci 2019, 15, 127-137.

11. Kim, S.H.; Juhnn, Y.S.; Song, Y.S. Akt involvement in paclitaxel chemoresistance of human ovarian cancer cells. Ann N Y Acad Sci 2007, 1095, 82-9.

12. Song, Y.;Li, L.;Ou, Y.;Gao, Z.;Li, E.;Li, X.;Zhang, W.;Wang, J.;Xu, L.;Zhou, Y. et al. Identification of genomic alterations in oesophageal squamous cell cancer. Nature 2014, 509, 91-5.

13. Li, B.;Xu, W.W.;Lam, A.K.Y.;Wang, Y.;Hu, H.F.;Guan, X.Y.;Qin, Y.R.;Saremi, N.;Tsao, S.W.;He, Q.Y. et al. Significance of PI3K/AKT signaling pathway in metastasis of esophageal squamous cell carcinoma and its potential as a target for anti-metastasis therapy. Oncotarget 2017, 8, 3875538766.

14. Liu, X.;Song, M.;Wang, P.;Zhao, R.;Chen, H.;Zhang, M.;Shi, Y.;Liu, K.;Liu, F.;Yang, R. et al. Targeted therapy of the AKT kinase inhibits esophageal squamous cell carcinoma growth in vitro and in vivo. Int J Cancer 2019,

15. Mori, M.; Kitazume, M.; Ose, R.; Kurokawa, J.; Koga, K.; Osuga, Y.; Arai, S.; Miyazaki, T. Death effector domain-containing protein (DEDD) is required for uterine decidualization during early pregnancy in mice. J Clin Invest 2011, 121, 318-27.

16. Zhang, S.;Chen, Q.;Liu, Q.;Li, Y.;Sun, X.;Hong, L.jJi, S.;Liu, C.;Geng, J.;Zhang, W. et al. Hippo Signaling Suppresses Cell Ploidy and Tumorigenesis through Skp2. Cancer Cell 2017, 31, 669684 e7.

17. Song, Q.; Zhen, H.; Liu, H.; Yuan, Z.; Cao, Z.; Zhao, B. A novel RhoA-related gene, DjRhoA, contributes to the regeneration of brain and intestine in planarian Dugesia japonica. Biochem Biophys Res Commun 2020, 533, 1359-1365.

18. Vainchenker, W.; Arkoun, B.; Basso-Valentina, F.; Lordier, L.; Debili, N.; Raslova, H. Role of RhoGTPases in megakaryopoiesis. Small GTPases 2021, 1-17. 
19. Geretto, M.; Pulliero, A.; Rosano, C.; Zhabayeva, D.; Bersimbaev, R.; Izzotti, A. Resistance to cancer chemotherapeutic drugs is determined by pivotal microRNA regulators. Am J Cancer Res 2017, 7, 1350-1371.

20. Thyagarajan, A.; Tsai, K.Y.; Sahu, R.P. MicroRNA heterogeneity in melanoma progression. Semin Cancer Biol 2019, 59, 208-220.

21. Lee, K.; Ferguson, L.R. MicroRNA biomarkers predicting risk, initiation and progression of colorectal cancer. World J Gastroenterol 2016, 22, 7389-401.

22. Kallioniemi, O.P.; Wagner, U.; Kononen, J.; Sauter, G. Tissue microarray technology for highthroughput molecular profiling of cancer. Hum Mol Genet 2001, 10, 657-62.

23. Friedlander, M.R.; Chen, W.; Adamidi, C.; Maaskola, J.; Einspanier, R.; Knespel, S.; Rajewsky, N. Discovering microRNAs from deep sequencing data using miRDeep. Nat Biotechnol 2008, 26, 407-15.

24. Wang, W.;Li, X.;Zhang, W.;Li, W.;Yi, M.;Yang, J.;Zeng, Z.;Colvin Wanshura, L.E.;McCarthy, J.B.;Fan, S. et al. Oxidored-nitro domain containing protein 1 (NOR1) expression suppresses slug/vimentin but not snail in nasopharyngeal carcinoma: Inhibition of EMT in vitro and in vivo in mice. Cancer Lett 2014, 348, 109-18.

25. Wang, W.; He, S.; Zhang, R.; Peng, J.; Guo, D.; Zhang, J.; Xiang, B.; Li, L. ALDH1A1 maintains the cancer stem-like cells properties of esophageal squamous cell carcinoma by activating the AKT signal pathway and interacting with beta-catenin. Biomed Pharmacother 2020, 125, 109940.

26. Wang, W.; Yi, M.; Chen, S.; Li, J.; Zhang, H.; Xiong, W.; Li, G.; Li, X.; Xiang, B. NOR1 Suppresses Cancer Stem-Like Cells Properties of Tumor Cells via the Inhibition of the AKT-GSK-3betaWnt/beta-catenin-ALDH1A1 Signal Circuit. J Cell Physiol 2017, 232, 2829-2840.

27. Krist, B.; Florczyk, U.; Pietraszek-Gremplewicz, K.; Jozkowicz, A.; Dulak, J. The Role of miR-378a in Metabolism, Angiogenesis, and Muscle Biology. Int J Endocrinol 2015, 2015, 281756.

28. Valmiki, S.; Ahuja, V.; Paul, J. MicroRNA exhibit altered expression in the inflamed colonic mucosa of ulcerative colitis patients. World J Gastroenterol 2017, 23, 5324-5332.

29. Zhu, Y.;Xiao, Y.;Kong, D.;Liu, H.;Chen, X.;Chen, Y.;Zhu, T.;Peng, Y.;Zhai, W.;Hu, C. et al. DownRegulation of miR-378d Increased Rab10 Expression to Help Clearance of Mycobacterium tuberculosis in Macrophages. Front Cell Infect Microbiol 2020, 10, 108.

30. Chen, J.; Niu, N.; Zhang, J.; Qi, L.; Shen, W.; Donkena, K.V.; Feng, Z.; Liu, J. Polyploid Giant Cancer Cells (PGCCs): The Evil Roots of Cancer. Curr Cancer Drug Targets 2019, 19, 360-367.

31. Zhang, S.; Mercado-Uribe, I.; Xing, Z.; Sun, B.; Kuang, J.; Liu, J. Generation of cancer stem-like cells through the formation of polyploid giant cancer cells. Oncogene 2014, 33, 116-28.

32. Zhang, D.; Yang, X.; Yang, Z.; Fei, F.; Li, S.; Qu, J.; Zhang, M.; Li, Y.; Zhang, X.; Zhang, S. Daughter Cells and Erythroid Cells Budding from PGCCs and Their Clinicopathological Significances in Colorectal Cancer. J Cancer 2017, 8, 469-478.

33. Niu, N.;Zhang, J.;Zhang, N.;Mercado-Uribe, I.;Tao, F.;Han, Z.;Pathak, S.;Multani, A.S.;Kuang, J.;Yao, J. et al. Linking genomic reorganization to tumor initiation via the giant cell cycle. Oncogenesis 2016, 5, e281.

34. Jiang, N.; Dai, Q.; Su, X.; Fu, J.; Feng, X.; Peng, J. Role of PI3K/AKT pathway in cancer: the framework of malignant behavior. Mol Biol Rep 2020, 47, 4587-4629.

35. Basant, A.; Glotzer, M. Spatiotemporal Regulation of RhoA during Cytokinesis. Curr Biol 2018, 28, R570-R580. 
